\title{
House dust mite immunotherapy in Germany: real-world adherence to a subcutaneous allergoid and a sublingual tablet
}

\author{
C. Vogelberg · B. Brüggenjürgen · H. Richter · M. Jutel
}

Received: 4 August 2020 / Accepted: 13 November 2020 / Published online: 17 December 2020

(C) The Author(s) 2020

\begin{abstract}
Summary
Purpose Allergen immunotherapy (AIT) is the only disease-modifying treatment for allergic rhinitis (AR) and asthma (AA) with increasing efficacy observed during the recommended 3 years of treatment and the potential for medium and long-term benefits. Adherence is of utmost importance to achieve these goals in real life. The objective of this study was to evaluate real-world adherence to house dust mite (HDM) AIT with a subcutaneous (SCIT) allergoid preparation and a sublingual (SLIT) tablet.

Patients and methods In this retrospective cohort analysis of a German longitudinal prescription database, patients receiving either a HDM-SCIT allergoid $(n=5677)$ or a HDM-SLIT tablet $(n=4720)$ were compared over a 3-year observation period. Study endpoints included adherence and days on therapy (DoT). Univariate statistical tests were used to assess the significance of the differences between SCIT vs. SLIT or the age classes.
\end{abstract}

Prof. Dr. C. Vogelberg ( $\bowtie)$

Department of Paediatric Pneumology and Allergology, University Hospital Carl Gustav Carus Dresden, Technical University Dresden, Fetscherstraße 74, 01307 Dresden, Germany

Christian.Vogelberg@uniklinikum-dresden.de

Prof. Dr. B. Brüggenjürgen

Institute for Health Service Research and Technical Orthopedics, Medical School Hannover, Hannover, Germany

Dr. H. Richter

Epidemiology, IQVIA, Frankfurt am Main, Germany

Prof. Dr. M. Jutel

All-MED Medical Research Institute, Wroclaw, Poland

Department of Clinical Immunology, Wroclaw Medical University, Wroclaw, Poland
Results Based on the number of reimbursed prescriptions SCIT adherence was $55.0 \%$ after 3 years while SLIT adherence was remarkably lower with $30.3 \%$ $(p<0.0001)$. The difference was observed already after first and second year of treatment and was in favor of SCIT with $93.2 \%$ vs. $63.2 \%$ and $70.9 \%$ vs. $43.4 \%$, respectively. After 3 years, days on therapy were significantly higher in the SCIT group with 824 days vs. 491 days $(p<0.0001)$ showing significantly better data for all age groups. Adherence to SCIT was the highest in children, followed by adolescents and adults while the adherence to SLIT was higher in adults than adolescents.

Conclusion In this retrospective real-world cohort analysis in Germany, patients on subcutaneous AIT for HDM respiratory allergy achieved significantly higher adherence rates compared to the HDM-SLIT tablet over a 3-year treatment period. In addition, days on therapy were significantly higher for SCIT than for SLIT. It was shown that in real life significantly more patients receiving SCIT stay on the recommended treatment period of 3 years compared to SLIT subjects. This is important since effectiveness has been demonstrated to be linked with therapy duration.

Keywords Adherence - Real-world evidence $\cdot$ House dust mite - Subcutaneous immunotherapy ·

Sublingual immunotherapy · Allergen

immunotherapy

Abbreviations

AA Allergic asthma

AR Allergic rhinitis

AIT Allergen immunotherapy

DoT Days on therapy

HDM House dust mites

QoL Quality of life 
RCT Randomized controlled trial

RWE Real-word evidence

SCIT Subcutaneous immunotherapy

SLIT Sublingual immunotherapy

WHO World Health Organization

\section{Introduction}

Respiratory allergic diseases including allergic rhinitis (AR) and allergic asthma (AA) demonstrate increase in prevalence rates worldwide and are underrecognized for their impact on health and daily living [1]. AR and AA can be considered as chronic diseases with potentially serious impact on patients' functioning and quality of life (QoL) and are associated with significant morbidity and extensive costs. AR affects the nose and possibly the eyes, resulting in a chronic, mostly eosinophilic, inflammation of the nasal mucosa and conjunctiva. It may be associated with sinusitis, hearing problems, and asthma. Even if there are no asthmatic symptoms at the beginning, AR can develop into AA over time. Thus, it is advisable not to treat only the symptoms and to provide short-time relief by pharmacotherapy but to alter the course of the disease $[2,3]$. The only causative treatment of AR and AA is AIT [2,4]. During AIT, the immune system develops tolerance to the triggering allergen due to the activation of allergen specific $\mathrm{T}$ regulatory cells subsets with the following desensitization measured by decreased IgE and increased IgG4. In addition to desensitization and thereby ameliorating symptoms, AIT also delivers long-term clinical benefits that may persist for years after discontinuation of the treatment and prevents the development of asthma [2, 5, 6]. Its efficacy in allergic rhinoconjunctivitis and asthma has been well established in several meta-analyses comprising many randomized controlled studies $[7,8]$.

In Europe, $26.5 \%$ of sensitized subjects suffer from a clinically relevant allergy to Dermatophagoides pteronyssinus, while between $11.4 \%$ in Austria and 63.5\% in Portugal are allergic to Dermatophagoides farinae [9]. Allergic conditions due to HDM may result in symptoms affecting the entire respiratory system causing respiratory allergic diseases. For AIT, both SCIT (subcutaneous immunotherapy) and SLIT (sublingual immunotherapy) preparations are available. According to current international and national guidelines, AIT should encompass at least 3 consecutive years [2, 4]. Adherence is a challenge for all treatments that should be taken or applied on a regular basis for a long period of time. There is a strong evidence that many patients with chronic disease experience difficulties in adherence to their recommended regimens. All over the world, nonadherence is a major problem to achieve a desired treatment outcome. According to the WHO, rates of nonadherence among patients with asthma range from $30 \%$ to $70 \%[10]$. Failure to adhere to a regular medication plan results in poor disease control with clinical consequences like worsening of symptoms, deterioration of the disease, decreased QoL and in economic consequences such as increased hospitalizations and waste of health care resources [10]. For AIT, adherence is of utmost importance, as lack of adherence is a contraindication, since the benefit of AIT depends on the duration of treatment $[2,4]$. When adhering to the recommended regimen, a reduced likelihood of developing asthma up to 2 years post AIT has been shown for pollen-allergic children and adolescents with AR after a 3-year treatment [2].

In contrast to randomized controlled trials (RCTs) with a clearly defined patient population and scheduled treatments, real-world evidence (RWE) data allow the investigation of real-life patients and their performance, making estimates of effectiveness including analysis of adherence possible [11, 12]. In order to obtain the adherence data, the objective of this RWE study was to analyze data from patients treated with allergoid HDM-SCIT and HDM-SLIT tablet over a period of 3 consecutive years with regard to medication adherence and analyze the days on therapy.

\section{Methods}

\section{Data source}

The analyses were carried out using the retrospective database IMS $^{\circledR} \operatorname{LRx}$ (IQVIA, Germany) which is based on reimbursed prescriptions of patients in Germany. The database covers about $82 \%$ of the statutorily insured population. Data are available at patient level (fully anonymized in accordance with German data protection legislation) and patients are trackable across pharmacies and physicians. The data include demographic information (sex, age) and all information related to prescriptions (e.g., prescription date, product, form, package size, package count, prescribing specialist). There is no information related to diagnoses in IMS ${ }^{\circledR}$ LRx.

\section{Overall analysis}

The overall analysis period was from January 2015 to December 2019. Patients with at least one prescription for either the HDM-SCIT allergoid (Acaroid ${ }^{\circledR}$, Allergopharma $\mathrm{GmbH} \&$ Co. KG, Reinbek, Germany) or the HDM-SLIT tablet (Acarizax ${ }^{\circledR}$, ALK-Abelló, Horsholm, Denmark) in the calendar year 2016 were selected, the first prescription that year being designated as the index prescription and its date as the index date. Patients were further validated for inclusion as being at least 5 years old (SCIT allergoid) or 12 years old (SLIT tablet) on the index date, not having had any previous mite AIT prescriptions and having had at least two focus prescriptions for one of the two products in the database. Prescription duration was imputed according to the manufacturer's recommendation and package size, and for the mite allergoid the 
Table 1 Patient counts and statistics for days on therapy by AIT product and age class

\begin{tabular}{lllll|lll}
\multicolumn{7}{c}{ HDM-SLIT tablet } & \multicolumn{7}{l}{ HDM-SCIT allergoid } \\
& Total & $12-17$ years & $18+$ years & Total & $5-11$ years & 12-17 years & $18+$ years \\
\hline $\begin{array}{l}\text { Total patient count } \\
\text { Days on therapy statistics }\end{array}$ & 4720 & 255 & 4465 & 5677 & 977 & 1049 & 3651 \\
\hline Mean & 491.4 & 397.5 & 496.7 & 823.7 & 883.4 & 828.4 & 806.4 \\
\hline Standard deviation & 330.5 & 301.1 & 331.4 & 271.1 & 259.8 & 269.5 & 272.2 \\
\hline Lower quartile & 180 & 120 & 180 & 597 & 777 & 630 & 567 \\
\hline Median & 438 & 271 & 446 & 927 & 995 & 927 & 898 \\
\hline Upper quartile & 817 & 669 & 823 & 1060 & 1080 & 1061 & 1050
\end{tabular}

package type (starter or maintenance) was additionally considered. From the prescription data, patients' adherence to focus therapy was calculated from the index until the first of the following events:

- Expiry of the last focus prescription

- Therapy pause between successive prescriptions (end of one to prescription of next) exceeding 9 months (274 days)

- Prescription of a nonfocus mite AIT product.

Since the maximum permitted pause between prescriptions was 9 months, it was not possible to state with acceptable certainty whether a patient whose therapy ended later than the 1 April 2019 (database end (31 December 2019) minus maximum therapy pause ( $=9$ months) $)$ had a true treatment cessation or might have continued giving a database update. In order to avoid a bias in the patients' composition after that date, the patients were not analyzed after this time point.

\section{Study endpoints}

\section{Adherence}

Adherence curves were calculated from the above information according to the Kaplan-Meier method for the period of up to 3 years (1095 days) after the index date. Separate curves were assessed for each product and, within product, for the index age classes (5-11 years, 12-17 years, 18+ years; the first of these only for the HDM-SCIT allergoid). Mean and median adherence was calculated from the curves.

\section{Days on therapy}

For the period of adherence, the total days on therapy were calculated by summing up the expected prescription durations of all focus prescriptions given within the adherence period. Duplicate days due to overlaps between prescriptions were reduced to single days and excess package reserve extending beyond the censoring date was not included in the analysis. Descriptive statistics (means, standard deviations, medians) were generated from the values thus obtained.

\section{Statistical testing}

For both adherence and days on therapy, the difference between the focus products and, within products, the age classes, were tested for statistical significance. For adherence, the curves were compared using a log-rank test whereas for days on therapy, due to the skewed distributions and observed heteroscedasticity (unequal scatter), nonparametric testing (Kruskal-Wallis test) was carried out. For both outcomes, the test was done on an all-against-all age class basis for the three age groups (mite allergoid), with a Bonferroni correction applied in each case to maintain the overall $p$-value for $H_{0}$ at 0.05 .

The software used for all analyses was SAS 9.4.

\section{Results}

A total of 10,397 patients were included in the analyses after all exclusion criteria had been applied, 5677 receiving the HDM-SCIT allergoid and 4720 the HDMSLIT tablet. Of these, $78 \%$ were adults, $22 \%$ adolescents and children aged 5-17 years (Table 1).

\section{Adherence}

The Kaplan-Meier curves (K-M curves) for adherence showed marked differences between the products that were in favor of the SCIT allergoid and statistically significant $(p<0.0001$, Fig. 1$)$. The SCIT product had a distinctly superior adherence even at the end of the first year of treatment $(93.2 \%$ were adherent to the HDM allergoid, $63.2 \%$ to the HDM tablet) that lasted through the second year $(70.9 \%$ vs. $43.4 \%$, respectively) to the end of the third year $(55.0 \%$ vs. $30.3 \%$, respectively).

The age class related pattern for adherence differed markedly between the two products. Whereas in SLIT patients, adults had a better adherence compared to adolescents (Fig. 2), adherence decreased with increasing age in the SCIT group (Fig. 3). For the HDM-SLIT tablet, $22.6 \%$ of adolescent patients were still adherent to the therapy after 3 years, whereas the same value was $30.7 \%$ in adults (Fig. 2). This difference developed gradually over the course of the adher- 
Fig. 1 Kaplan-Meier curves for adherence in patients receiving HDM-SCIT with allergoid $(n=5677)$ or HDMSLIT with a tablet $(n=4720)$ with the results of the logrank comparisons between the curves

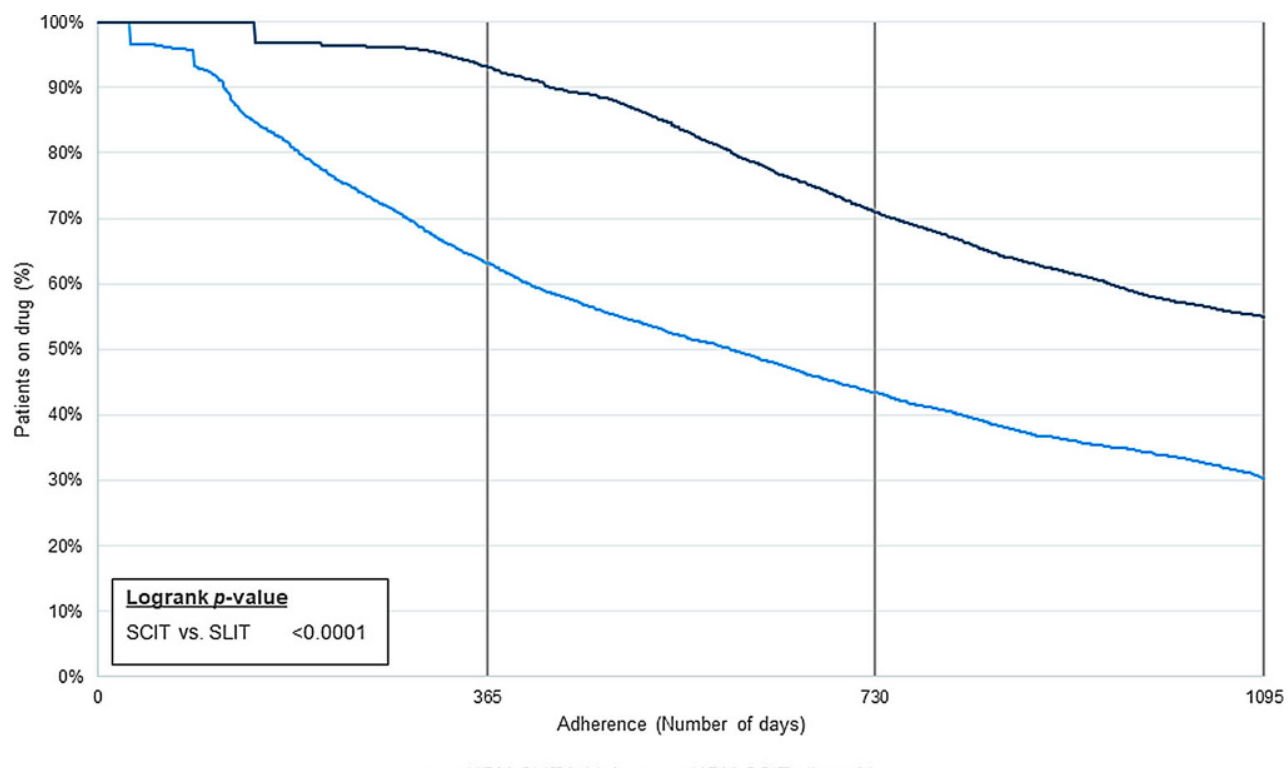

Fig. 2 Kaplan-Meier curve for adherence in patients receiving AIT with the HDMSLIT tablet by age class with the results of the log-rank comparisons between curves $(n=255$, 12-17 years; $n=4465$, $\geq 18$ years)

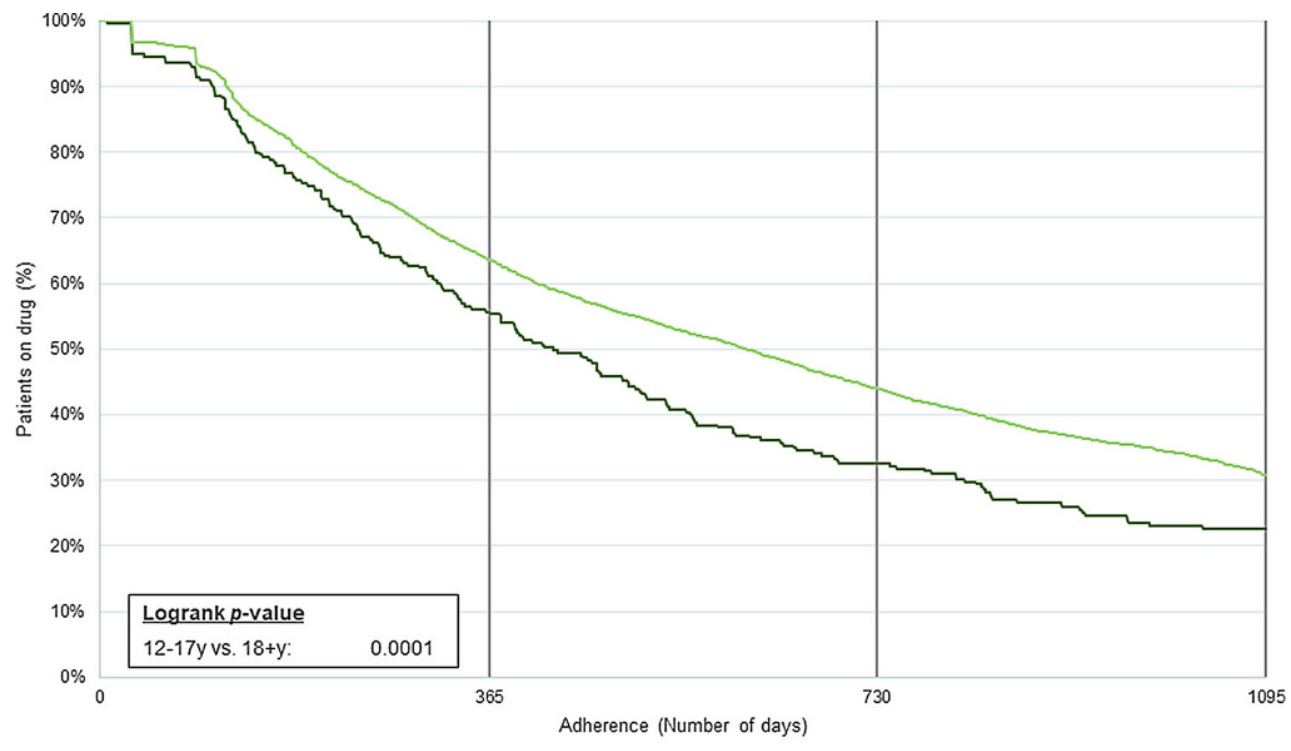

-12-17 years - $18+$ years

ence curve and was statistically significant ( $p=0.0001$, Fig. 2).

In the SCIT group, on the other hand, adherence rates ranged between $93.0 \%$ and $93.8 \%$ for all age groups at the end of the first year showing almost no difference. Thereafter, they diverged gradually. At the end of the third year, $68.5 \%$ of children, $57.6 \%$ of adolescents and $50.6 \%$ of adults remained adherent. All differences were significant ( $p<0.0001$ for all comparisons, Fig. 3).

\section{Days on therapy}

The results for DoT largely reflected those of the adherence analysis (Table 1, Fig. 4). Patients being treated with the SLIT tablet had a lower mean
DoT value (491 days) than those in the SCIT group (824 days). Adolescents receiving SLIT (398 days) had almost 100 fewer days on therapy than the respective adults (497 days). For the SCIT allergoid, the age class differences were obviously lower ranging from 806 to 883 DoT (Fig. 4). All comparisons were found to be significant ( $p=0.0344$ for SCIT allergoid adults vs. adolescents, $p<0.0001$ for all other comparisons, Fig. 4).

\section{Discussion}

Allergic diseases may cause not only severe symptoms but potentially have a serious impact on patients' overall health status and quality of life [1, 3]. In contrast to symptomatic treatment, AIT not only 
Fig. 3 Kaplan-Meier curves for adherence in patients receiving AIT with the HDMSCIT allergoid by age class with the results of the log-rank comparisons between the curves $(n=977$, 5-11 years; $n=1049$, 12-17 years; $n=3651$, $\geq 18$ years)

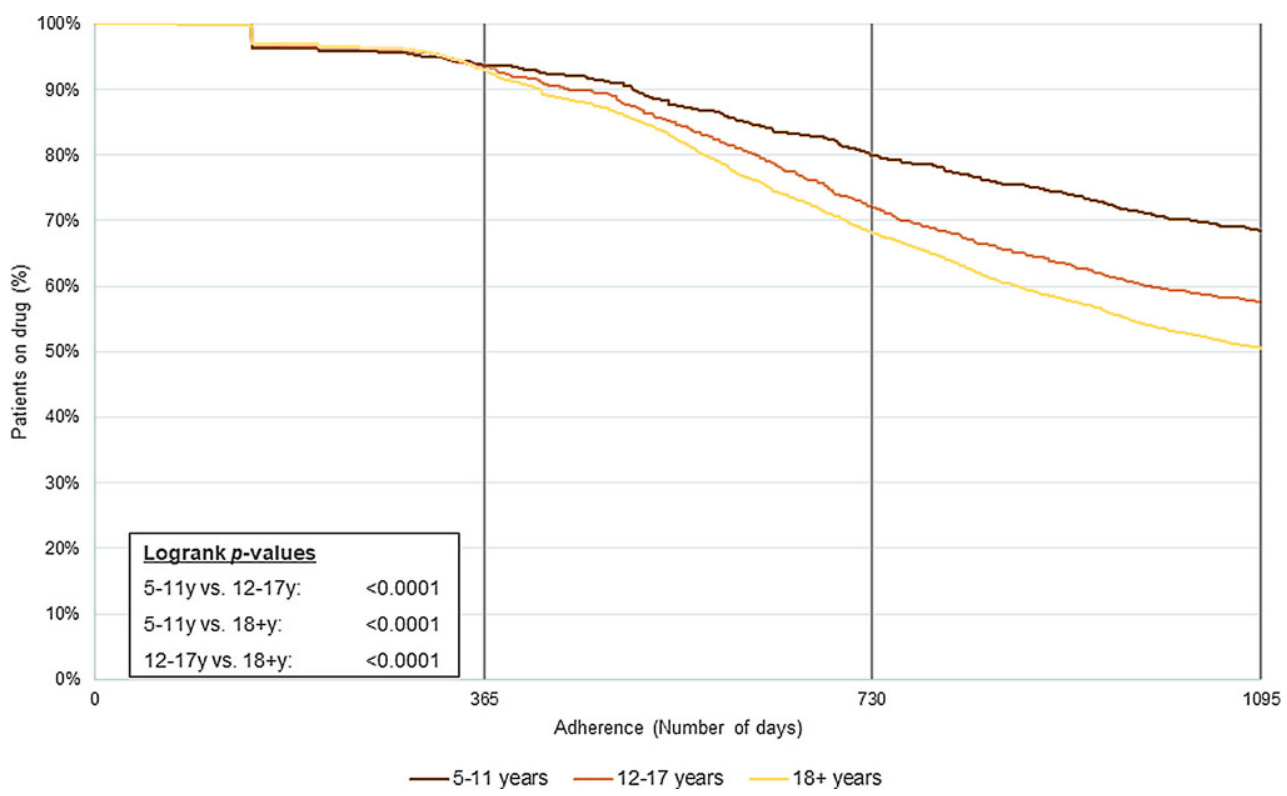

Fig. 4 Days on therapy for the HDM-SCIT with an allergoid and HDM-SLIT with a tablet. a The HDM SLIT tablet has no marketing authorization for children

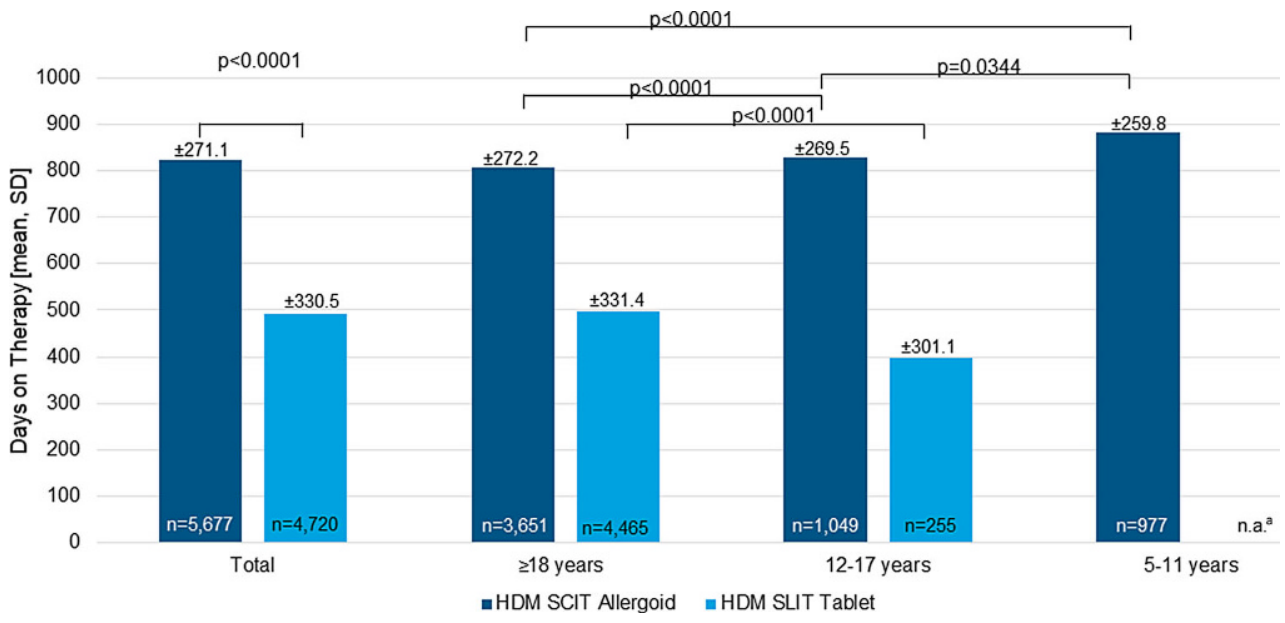

ameliorates the symptoms but in addition has the capacity to change the natural course of disease and achieve long-term clinical benefits after termination of AIT [2, 3]. National and international guidelines recommend administering of AIT for at least 3 years $[2,4]$. In order to achieve the long-term efficacy, medication adherence is paramount because the success of AIT depends on the duration of treatment. Therefore, it is particularly important that AIT is carried out in accordance with the prescriber's recommendations $[4,13]$. However, adherence is a challenge in chronic diseases especially if the disease is considered not very serious like it is often the case in allergy. It was shown that the greater the perceived disease severity the better drug compliance [14]. Medication adherence is defined as an active, cooperative and voluntary participation of the patient on following recommendations from a healthcare provider, e.g., regarding timing, frequency and dosage [15]. RWE analyses are an ideally suited study model because they provide information on treatment practices in specific populations without a predetermined study protocol. As the efficacy of a medication is directly influenced by its adherence in daily life, RWE data offer valuable clues towards effectiveness in addition to the efficacy proven in RCTs (socalled maximum efficacy). This maximum efficacy is modified in two steps when transferring the treatment into real life. The first step is "from bench to bedside" and the efficacy is reduced due to transfer and implementation losses in hospitals. In the second step "from bedside to routine care" a further reduction in efficacy is seen when another loss in implementation is added to the previously mentioned challenges in daily routine care. These steps lead from the maximum efficacy in RCTs to a downscaled effectiveness in real life where adherence plays a major role [16].

The aim of this controlled retrospective RWE study was to investigate adherence to AIT over a period of 3 years. The patients were treated with two different HDM AIT preparations, either a subcutaneous HDM 
allergoid or a sublingual HDM tablet. The preparations were chosen because at the time of the index period both products were market leaders in their respective segment (HDM-SCIT or HDM-SLIT; independent data from IMS ${ }^{\circledR}$ Pharmascope, IQVIA, MAT 12/2016). At the end of the third year, adherence for both SCIT and SLIT was not as high as recommended and reflects the common challenge of adherence for long-term treatments [10]. Nevertheless, the overall adherence for the recommended 3-year treatment period for SCIT was significantly higher than for SLIT. The Kaplan-Meier curves as a demonstration for time-dependent events show favorable data for SCIT vs. SLIT already in year one and two until the end of the third year $(55.0 \%$ vs. $30.3 \%$, respectively). More than a third had discontinued SLIT yet after the first year.

The observed adherence rates in our analysis are generally similar to the results of previous studies across Europe with a slight trend towards higher values $[17,18]$. In the present study, adherence was defined as the time from the first focus prescription to the expiry of the last focus prescription. Using the same definition, another German RWE data analysis by Jutel et al. using the same SCIT allergoid led to different results: adherence after year two (63.8\%) and year three $(38.6 \%)$ was considerably lower than in the present study [18]. Though investigating the same HDM SCIT preparation, the data are not directly comparable due to the different approaches used. Jutel et al. investigated data from 2008-2017 comprising 4 years after the index prescription while this data analysis covers the years 2015-2019 with an observation period of 3 years after the index prescription.

When analyzing different pollen SCIT and SLIT products in our previous RWE analysis, we showed lower results for SCIT than in this study with nearly $61 \%$ after year two and approximately $36 \%$ after three years. For SLIT (investigating both tree pollen drops and grass pollen tablets), the values were even lower than in this analysis with a range from $29.6 \%$ to $36.9 \%$ and merely $9.6 \%$ to $18.2 \%$ after year two and three, respectively. Different from HDM AIT adherence our previous analysis of grass and pollen AIT adherence covers pre-, pre-coseasonally or perennially administration schemes which contrasts with HDM AIT being administered perennially only. Therefore, despite the same definition of adherence different SCIT and SLIT outcomes for HDM vs. pollen may be possible.

The described patient population included adolescents and adults for the SLIT group while the SCIT group additionally comprised children. The reason for this difference is that the SLIT tablet is not approved for children while the SCIT product can be administered starting at the age of 5 years. Splitting the results into different age groups showed the best adherence in children followed by adults and adolescents when being treated with the SCIT allergoid. Previous analyses showed the same adherence trend during SCIT for the different age groups as in our analysis with children being more adherent than adults and adolescents [18-20]. This may be explained by the parental care wanting the best for their children and thus being very consistent with the implementation of the treatment plan. Adults often prioritize other things especially when considering an allergy as a nonserious disease and neglect the treatment. In line with other studies the outcome of this study demonstrates that adherence depends on the patient's mindset and that guidance either from HCPs or parents can have significant influence on it [10, 21, 22].

In general, published data indicate that nonadherence is more common among adolescents [23]. Medication adherence in adolescents is low even when suffering from chronic diseases which demand regular drug use like multiple sclerosis [24], diabetes [25] or epilepsy [26, 27]. Adolescents have a sense of exceptionalism and immortality [25], are looking for freedom, being independent, tending to trivialize their disease(s) and/or not willing to accept any discomfort from adverse events. This might be the reason for significantly less adherence to the self-administered SLIT than in adults because of the inconvenience of taking the tablets every day. Available evidence suggests that adolescents and parents disagree on who is primarily responsible for medication use. Physician involvement and frequency of interaction between the patients and physician aids to better adherence [20, 28] which might be the reason that adherence in adolescents is higher to SCIT (57.6\%) than to SLIT (22.6\%) in the analysis at hand.

No need to visit the doctor's office regularly is in favor of SLIT which can be administered at home. Doctor's consultancy takes place on a quarterly basis only which interferes less with the patient's daily life. But it seems that the regular contact between physician and patient has a favorable influence on adherence. This was already discussed in an Italian prospective study investigating SLIT adherence in children and adolescents over 3 years in relation to the frequency of doctor's visits. The group with regular visits showed significantly lower withdrawal rates than the groups with fewer visits. The authors concluded that SLIT should be monitored frequently from the beginning to the end of treatment to check the effective co-operation of patients [29]. Thus, the above-mentioned advantage for SLIT cannot be fully supported, whereas SCIT seems to fulfill the criteria which enhance adherence.

The calculations of the days on therapy (DoT) underline the better adherence data over time for SCIT in comparison to SLIT. These results are in line with other German RWE data where SCIT also showed considerably greater DoT than SLIT [19]. Though our previous study investigated adherence of pollen-allergic patients, the trend is the same with higher absolute values than in this analysis because of the different methodology. In our first study, we examined a period of 8 years while in this study the observation 
was limited to 3 years. The reason for this is that the SLIT tablet gained market access at the end of 2015 and therefore, it was not possible to prolong surveillance. Hence, this study provides the first real-world evidence data for the HDM-SLIT tablet.

Adherence rates from RCTs cannot be transferred into real life as the setting is completely different. In addition to a strict protocol, patients are often supervised by study nurses and might be paid for their participation. These measures have a strong influence on adherence and do not reflect daily life. Thus, reported adherence rates show a great variance with ranges from $18 \%$ to over $90 \%$ and were higher in clinical studies than real-life surveys with overlapping ranges for SCIT and SLIT [2]. This present study confirms that adherence in real life is generally lower than in controlled studies.

Depending on selected methods, definitions and parameters, results of "real life" adherence studies extremely vary and are not directly comparable. Sieber et al. assessed medication adherence using prescription renewal rates for grass pollen AIT in a representative population of patients in Germany to evaluate whether the perception of superior adherence for the subcutaneous route compared to the sublingual route could be confirmed in clinical practice [30]. Contradictory to our results the group observed significantly higher adherence rates for SLIT than for SCIT. The method was based on the simple calculation of the number of packs ignoring the range of coverage which probably influenced the results [30]. For our analysis, in addition to the number of packs, each pack size was considered and its days of coverage were calculated. This incremental difference gives a more realistic view compared to other studies [20,30].

Several factors influence medication adherence and they are weighted differently [22, 31]. Patient-, physician- and therapy-related factors seem to be important, overlapping with social parameters. These observations are in line with the WHO and its definition of five dimensions that are essentially influencing adherence [10]: (1) Social and economic factors; (2) Health care team and system-related factors; (3) Conditionrelated factors; (4) Therapy-related factors, and (5) $\mathrm{Pa}-$ tient-related factors. Thus, adherence is a complex construct with variable factors that need to be adapted to the particular circumstances. The guideline recommendation of at least 3 years of AIT duration is probably easier to achieve with SCIT than with SLIT in real life.

The present study bares some limitations which arose from the fact that secondary data was used for the analyses resulting in unknown physician's recommendation as to the posology of the medication investigated. For the HDM tablet, this is irrelevant since the posology is clearly defined by the manufacturer and it is highly unlikely that the doctor intends the patient to deviate from it. For the HDM allergoid, there is some variability with respect to the intervals be- tween maintenance injections ("4 to 8 weeks") so that only average values could be applied. Since the permitted gap of 9 months between prescriptions in the adherence analysis will easily have sufficed to bridge any gap arising from underestimation of prescription duration and since any prescription overlap was eliminated in both analyses (adherence and days on therapy), this limitation is expected to have had a minor effect on the results.

Another limitation of this retrospective analysis includes the assessment of reimbursed prescriptions from the German statutory health insurance (GKV). The actual number of SCIT injections or intake of SLIT tablets cannot be extracted from such data. Such data can only be obtained from randomized controlled trials with strict monitoring and documentation procedures. However, the commonly high adherence in clinical trials does not mirror the daily behavior of patients in real life treatment but are the result from strict management follow-up according to study plans [2]. This is why we choose the well-established design approach based on GKV prescription data using the coverage data given in the SmPCs of the preparations as base for calculation of adherence [18, 19, 32]. Another aspect of uncertainty may originate from patients' varying drug intake compared to the original prescription not taking the drug as prescribed [33]. However, this might be more relevant for the SLIT with tablets administered at home.

\section{Conclusion}

The data from this retrospective cohort adherence analysis of a German longitudinal prescription database revealed the challenge to follow the guidelinerecommended treatment duration of 3 years. During the observation period, the analysis showed a higher adherence for patients treated with a HDM-SCIT allergoid compared to a HDM-SLIT tablet and a significantly higher number of days on therapy in the HDM-SCIT allergoid treated patients. More effective tools for support and increase of adherence in AIT are needed.

Acknowledgements This study was funded by Allergopharma GmbH \& Co. KG, Reinbek, Germany.

Author Contribution Hartmut Richter contributed to the design of the study, performed the statistical analyses, and corrected the manuscript. Christian Vogelberg, Marek Jutel and Bernd Brüggenjürgen contributed to the design of the study and corrected the manuscript. Katrin Engemann, SPM PharmaBusiness, Hamburg, managed the literature searches and wrote the first draft of the manuscript.

Funding Open Access funding enabled and organized by Projekt DEAL.

Conflict of interest C. Vogelberg has received lecture or consulting fees from ALK-Abelló, Allergopharma, Allergy Therapeutics, AstraZeneca, Boehringer Ingelheim, Bencard Allergy, DBV Technologies, LETI Pharma, Novartis Pharma and 
Sanofi Aventis. M. Jutel has received lecture or consulting fees from ALK-Abelló, Allergopharma, Stallergenes, Anergis, Allergy Therapeutics, Circassia, LETI Pharma, Biomay, HAL Allergy, Astra-Zeneca, GSK, Novartis, Teva, Vectura, UCB, Takeda, Roche, Janssen, Medimmune, and Chiesi. B. Brüggenjürgen reports grants from Allergopharma during the conduct of the study. $\mathrm{H}$. Richter declares that he has no competing interests. The authors have no other relevant affiliations or financial involvement with any organization or entity with a financial interest in or financial conflict with the subject matter or materials discussed in the manuscript apart from those disclosed.

Open Access This article is licensed under a Creative Commons Attribution 4.0 International License, which permits use, sharing, adaptation, distribution and reproduction in any medium or format, as long as you give appropriate credit to the original author(s) and the source, provide a link to the Creative Commons licence, and indicate if changes were made. The images or other third party material in this article are included in the article's Creative Commons licence, unless indicated otherwise in a credit line to the material. If material is not included in the article's Creative Commons licence and your intended use is not permitted by statutory regulation or exceeds the permitted use, you will need to obtain permission directly from the copyright holder. To view a copy of this licence, visit http://creativecommons.org/licenses/by/4.0/.

\section{References}

1. Pawankar R, Canonica GW, Holgate ST, Lockey RF, editors. WAO white book on allergy. Milwaukee: World Allergy Organization; 2011.

2. Roberts G, Pfaar O, Akdis CA, Ansotegui IJ, Durham SR, van Gerth Wijk R, et al. EAACI guidelines on allergen immunotherapy: allergic rhinoconjunctivitis. Allergy. 2018;73:765-98. https://doi.org/10.1111/all.13317.

3. Bousquet J, KhaltaevN, CruzAA, DenburgJ, Fokkens WJ, Togias A, et al. Allergic rhinitis and its impact on asthma (ARIA) 2008 update (in collaboration with the World Health Organization, GA(2)LEN and AllerGen). Allergy. 2008;63(Suppl 86):8-160. https://doi.org/10.1111/j.13989995.2007.01620.x.

4. PfaarO, BachertC, BufeA, BuhlR, EbnerC,EngP, etal. Guideline on allergen-specific immunotherapy in IgE-mediated allergic diseases: S2k guideline of the German Society for Allergology and Clinical Immunology (DGAKI), the Society for Pediatric Allergy and Environmental Medicine (GPA), the Medical Association of German Allergologists (AeDA), the Austrian Society for Allergy and Immunology (ÖGAI), the Swiss Society for Allergy and Immunology (SGAI), the German Society of Dermatology (DDG), the German Society of Oto- Rhino-Laryngology, Head and Neck Surgery (DGHNO-KHC), the German Society of Pediatrics and Adolescent Medicine (DGKJ), the Society for Pediatric Pneumology (GPP), the German Respiratory Society (DGP), the German Association of ENT Surgeons (BV-HNO), the Professional Federation of Paediatricians and Youth Doctors (BVKJ), the Federal Association of Pulmonologists (BDP) and the German Dermatologists Association (BVDD). Allergo J Int. 2014;23:282-319. https://doi.org/10.1007/ s40629-014-0032-2.

5. JacobsenL, NiggemannB, DreborgS, FerdousiHA, HalkenS, Høst A, et al. Specific immunotherapy has long-term preventive effect of seasonal and perennial asthma: 10-year follow-up on the PAT study. Allergy. 2007;62:943-8. https:// doi.org/10.1111/j.1398-9995.2007.01451.x.

6. Durham SR, Emminger W, Kapp A, de Monchy JGR, Rak S, Scadding GK, et al. SQ-standardized sublingual grass immunotherapy: confirmation of disease modification 2 years after 3 years of treatment in a randomized trial. J Allergy Clin Immunol. 2012;129:717-725.e5. https:// doi. org/10.1016/j.jaci.2011.12.973.

7. Dhami S, Kakourou A, Asamoah F, Agache I, Lau S, Jutel M, et al. Allergen immunotherapy for allergic asthma: a systematic review and meta-analysis. Allergy. 2017;72:1825-48. https://doi.org/10.1111/all.13208.

8. Dhami S, Nurmatov U, Arasi S, Khan T, Asaria M, Zaman H, et al. Allergen immunotherapy for allergic rhinoconjunctivitis: a systematic review and meta-analysis. Allergy. 2017;72:1597-631. https://doi.org/10.1111/all.13201.

9. Burbach GJ, Heinzerling LM, Edenharter G, Bachert C, Bindslev-Jensen C, Bonini S, et al. GA(2)LEN skin test study II: clinical relevance of inhalant allergen sensitizations in Europe. Allergy. 2009;64:1507-15.

10. World Health Organization. Adherence to long-therm therapies: evidencefor action. Geneva: WHO;2003.

11. OyinlolaJO, Campbell J, Kousoulis AA. Is realworld evidence influencing practice? A systematic review of CPRD research in NICE guidances. BMC Health Serv Res. 2016;16:299. https://doi.org/10.1186/s12913-016-1562-8.

12. Khosla S, White R, Medina J, Ouwens M, Emmas C, Koder T, et al. Real world evidence (RWE) - a disruptive innovation or the quiet evolution of medical evidence generation? F1000Res. 2018;7:111. https://doi.org/10.12688/ f1000research.13585.1.

13. Klimek L, Bachert C, Pfaar O, Becker S, Bieber T, Brehler R, et al. ARIA guideline 2019: treatment of allergic rhinitis in the German health system. Allergo J Int. 2019;28:255-76. https://doi.org/10.1007/s40629-019-00110-9.

14. DiMatteo MR, Haskard KB, Williams SL. Health beliefs, disease severity, and patient adherence: a meta-analysis. Med Care. 2007;45:521-8. https://doi.org/10.1097/MLR. 0b013e318032937e.

15. CramerJA, BenedictA, MuszbekN, KeskinaslanA, KhanZM. The significance of compliance and persistence in the treatment of diabetes, hypertension and dyslipidaemia: a review. Int JClin Pract. 2008;62:76-87.

16. Rebscher H, Kaufmann S, editors. Gesundheitssysteme im Wandel. Heidelberg, München, Landsberg, Frechen, Hamburg: Economica; 2009.

17. Kiel MA, Röder E, van Gerth Wijk R, Al MJ, Hop WCJ, Rutten-van Mölken MPMH. Real-life compliance and persistence among users of subcutaneous and sublingual allergen immunotherapy. J Allergy Clin Immunol. 2013;132:353-360.e2. https://doi.org/10.1016/j.jaci.2013. 03.013 .

18. Jutel M, Brüggenjürgen $B$, Vogelberg $C$, Richter H. Realworld evidence of subcutaneous allergoid immunotherapy in house dust mite-induced allergic rhinitis and asthma. Allergy. 2020;75:2046-54. https://doi.org/10.1111/all. 14240 .

19. Vogelberg C, Brüggenjürgen B, Richter H, Jutel M. Realworld adherence and evidence of subcutaneous and sublingual immunotherapy in grass and tree pollen-induced allergic rhinitis and asthma. Patient Prefer Adherence. 2020;14:817-27. https://doi.org/10.2147/PPA.S242957.

20. Egert-Schmidt A-M, Kolbe J-M, Mussler S, Thum-Oltmer S. Patients' compliance with different administration routes for allergen immunotherapy in Germany. Patient Prefer Adherence. 2014;8:1475-81. https://doi.org/10.2147/PPA. S70326. 
21. Kardas P, LewekP, Matyjaszczyk M. Determinants of patient adherence: a review of systematic reviews. Front Pharmacol. 2013;4:91. https://doi.org/10.3389/fphar.2013. 00091

22. Senna G, Caminati M, Lockey RF. Allergen immunotherapy adherence in the real world: How bad is it and how can it be improved? Curr Treat Options Allergy. 2015;2:39-53.

23. Schaefer MR, Kavookjian J. The impact of motivational interviewing on adherence and symptom severity in adolescents and young adults with chronic illness: a systematic review. PatientEduc Couns. 2017;100:2190-9.

24. Thannhauser JE, Mah JK, Metz LM. Adherence of adolescents to multiple sclerosis disease-modifying therapy. Pediatr Neurol. 2009;41:119-23.

25. Borus JS, Laffel L. Adherence challenges in the management of type 1 diabetes in adolescents: prevention and intervention. Curr Opin Pediatr. 2010;22:405-11.

26. Smith AW, Mara CA, Modi AC. Adherence to antiepileptic drugs in adolescents with epilepsy. Epilepsy Behav. 2018;80:307-11.

27. Carbone L, et al. Treatment adherence among adolescents with epilepsy: What really matters? Epilepsy Behav. 2013;27:59-63.

28. Holbein CE, et al. Allocation of treatment responsibility in adolescents with epilepsy: associations with cogni- tive skills and medication adherence. J Pediatr Psychol. 2019;44:72-83.

29. Vita D, Caminiti L, Ruggeri P, Pajno GB. Sublingual immunotherapy: adherence based on timing and monitoring control visits. Allergy. 2010;65:668-9. https://doi.org/10. 1111/j.1398-9995.2009.02223.x.

30. Sieber J, de Geest S, Shah-Hosseini K, Mösges R. Medication persistence with long-term, specific grass pollen immunotherapy measured by prescription renewal rates. Curr Med Res Opin. 2011;27:855-61. https://doi.org/10. 1185/03007995.2011.559538.

31. Reisacher WR, Visaya JM. Patient adherence to allergy immunotherapy. Curr Opin Otolaryngol Head Neck Surg. 2013;21:256-62. https://doi.org/10.1097/MOO. 0b013e32835f8048.

32. Allam J-P, Andreasen JN, Mette J, Serup-Hansen N, Wüstenberg EG. Comparison of allergy immunotherapy medication persistence with a sublingual immunotherapy tablet versus subcutaneousimmunotherapyin Germany. JAllergy Clin Immunol. 2018;141:1898-1901.e5.

33. Galozy A, Nowaczyk S. Prediction and pattern analysis of medication refill adherence through electronic health records and dispensation data. J Biomed Inform X. 2020;6-7:100075 